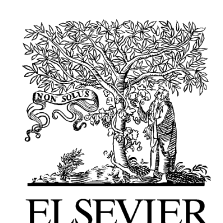

\title{
LETTER FROM THE EDITOR
}

\section{Is Treating all Pressure Wound Patients with Vitamins A, C, Zinc and Arginine Justified?*}

Vitamins are integral part of our daily diet. Healthy individuals and even patients who are eating healthy diet will ingest enough vitamins and supplements to meet their needs. Vitamins and zinc are important elements in wound healing hence severely malnourished patients will have vitamins and zinc deficiency. Extreme cases of scurvy and other severe vitamins deficiencies is rarely seen in the US but are still encountered in the developing countries.

In general, the nutrition status of the patient may correlate with the levels of albumin, pre albumin, transferrin and other serum proteins with the exception of patients on steroids, with severe sepsis, with systemic inflammatory disease or on dialysis. Of course the clinical picture including hydration, proteins intake and calorie counts, weight variations are important to assess.

The current trend of managing patients with pressure wounds includes the administration of high doses of vitamins $\mathrm{A}$ and $\mathrm{C}$, Zinc and Arginine without even checking their serum levels. To date there are no high evidencedbased studies (Prospective Randomized Controlled Trials) to prove the benefits of this standard. In addition to increasing the cost of healthcare, some of these vitamins may have toxicity and side effects that can be avoided by not prescribing them unnecessarily.

Vitamin $\mathrm{C}$ toxicity may produce diarrhea, nausea, may increase the risk for bleeding and formation of kidney stones. Vitamin A toxicity includes changes to vision, bone pain, skin changes, dizziness and in the chronic exposure, patients may have liver damage and coma. Zinc toxicity includes abdominal pain, nausea, vomiting, dizziness, lethargy and anemia, cramps headache and brain toxicity. Arginine toxicity may cause abdominal pain, bloating, diarrhea, worsening of asthma, low blood pressure.

Unfortunately, some healthcare providers who work for plaintiff attorneys rush to the conclusion of "deviating from standard of care" in variety of wound care cases, mainly pressure wounds, where vitamins and zinc were not prescribed without even verifying their serum levels. They assume, based on speculation only, that not placing all these patients on mega dose of vitamins and zinc, is deviation from the standard of care and that the levels on admission were low. This approach may hurt some patients due to toxicity, increases the number of frivolous lawsuits, and increases the cost of health care and the financial burden on the system. The high cost of litigation may channel some of the money that otherwise should have been spent on patients care and spends it on lawsuits with no merit.

By Richard Simman, MD, FACS, FACCWS

Department of Pharmacology and Toxicology, Wright State University Boonshoft School of Medicine, Dayton, OH, USA

Department of Dermatology, Wright State University Boonshoft School of Medicine, Dayton, OH, USA

\section{E-mail address:richard.simman@wright.edu}

* This letter represents the opinion of the author. 